\title{
Genetic Analysis of Grain Yield and Its Attributes Using Diverse Systems of Male Sterile Lines and Pollinators in Direct Sown and Ratooned Pearl Millet
}

\author{
Ramesh Kumar*, Sudhir Sharma, Prem Sagar and Ashok Kumar
}

Department of Plant Breeding, CCS Haryana Agricultural University, Hisar, India

*Corresponding author

\section{Ke y w ords \\ Combing ability, Cytoplasm, Male sterility, gca effects, sca effects, \\ Environment, Rationing, Pearl millet}

Article Info

Accepted:

05 January 2020

Available Online:

10 February 2020

\section{A B S T R A C T}

The genetics of grain yield and its attributes were studied using diverse cytoplasmic male sterile lines in 144 hybrids developed by crossing six A- and their six B-lines with 12 R-lines of pearl millet in a line $x$ tester mating design. The six $A$ - lines $81 \mathrm{~A}_{1}$ and $8 \mathrm{~A}_{1}\left(\mathrm{~A}_{1}\right), \mathrm{Pb} 313 \mathrm{~A}\left(\mathrm{~A}_{2}\right), \mathrm{Pb} 402 \mathrm{~A}\left(\mathrm{~A}_{3}\right), 81 \mathrm{~A}_{4}$ and $81 \mathrm{~A}_{5}$ and their corresponding $\mathrm{B}$ - lines represented five different systems of male sterility were very diverse. The 24 parents and the 144 hybrids were grown separately in contiguous blocks in $2 \mathrm{r} x$ $2.5 \mathrm{~m} \times 0.45 \mathrm{~m}$ in randomized block design with two replications in six environments under natural condition viz., early sown non-cut crop $\left(\mathrm{E}_{1}, \mathrm{E}_{4}\right)$, ratoon crop $\left(\mathrm{E}_{2}, \mathrm{E}_{5}\right)$ and late sown non-cut crop $\left(\mathrm{E}_{3}\right.$, $\left.\mathrm{E}_{6}\right)$ at Research Farm, CCSHAU, Hisar. The analysis of variance for combining ability revealed that the mean squares due to lines, testers and lines $\times$ testers were highly significant when tested against the error mean sum of squares for all the characters in all the environments during both years. The higher magnitude of fixed effect sca variances to that of fixed effect gca variances for all the characters indicated that non-additive gene effects governed the inheritance. The perusal of data with respect to gca effects of lines revealed that 3 (313A) and 9 (313B) representing $\mathrm{A}_{2}$ system of male sterile cytoplasm and fertile cytoplasm combined favourably for grain yield and growth rate, exhibited significant positive gca effects in most of environment including ratoon crop. Lines 4 (402A) and $10(402 \mathrm{~B})$ of $\mathrm{A}_{3}$ system proved good general combiners for 500 grain weight. Lines 5 $\left(81 \mathrm{~A}_{4}\right.$ and $\left.81 \mathrm{~B}_{4}\right)$ of $\mathrm{A}_{4}$ system showed significant positive gca effects for harvest index, turned to be good general combiner. Tester 17 (77/245) emerged to be good general combiner for grain yield, harvest index and growth rate, exhibited significant positive gca effects in most of environments for these characters. Other testers showed significant positive gca effects for grain yield in most of the environments were 20 (ICR 161), 16 (CSSC46-2) and 23 (77/180). Tester 20 (ICR 161) was also good general combiner for 500 grain weight. The hybrids $1 \times 13\left(81 \mathrm{~A}_{1} \times \mathrm{H} 90 / 4-5\right), 1 \times 14\left(81 \mathrm{~A}_{1} \times\right.$

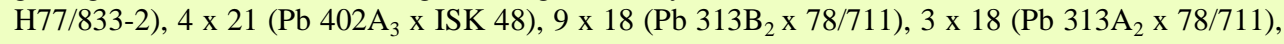
$11 \times 18\left(81 B_{4} \times 78 / 711\right), 12 \times 16\left(81 B_{5} \times\right.$ CSSC 46-2), 7 × $21\left(81 B_{1} \times\right.$ ISK 48$)$ showed significant positive sca effects for grain yield and one or more characters in most of the entrainments. The hybrids 1 x $13\left(81 \mathrm{~A}_{1}\right.$ x H90/4-5), 1 x $14\left(81 \mathrm{~A}_{1}\right.$ x H77/833-2), 9 × $13\left(313 \mathrm{~B}_{2}\right.$ × H90/4-5) and 12 × 16 $\left(81 \mathrm{~B}^{5} \times\right.$ CSSC46-2) performed better on the basis of per se, sca effects including ratoon crop indicating their hope for sustainable dual purpose pearl millet production in harsh environments and can be recombined to develop base population for improvement for ratoonability by cyclic breeding. 


\section{Introduction}

Pearl millet (Pennisetum glaucum (L) R. Br.), an important food, feed and dual purpose crop, is life line of human and cattle population inhabiting under most trying conditions in India and Africa. The crop is cultivated mainly under poor fertility, low inputs, severe heat and rainfed conditions where drought in one to four years is not uncommon. It assumes greater significance as no other crop competes with its biomass production potential and per day productivity. Green fodder of pearl millet is preferred over that of sorghum because its HCN content is low and can be fed to cattle without harm at any stage of growth Unavailability of green fodder in pre-monsoon periods is frequent therefore, identification of lines for regeneration potential and with ability to give good grain yield holds significance. The appreciable higher grain yield of pearl millet in crop cut after 35 days of sowing in comparison to non-cut reported by Tomer and Saini (1979) is encouraging. Day et al., (1968) and Skorda (1977) also reported increase in grain yield in cereal crops after cut. Kumar et al., (2005) observed that regeneration and ratooning ability under genetic control and regeneration potential could be incorporated in genotype with high grain yield in pearl millet. Thus, the practice of sowing the crop early sometimes in June to reduce the risk of non-receipt of rains in time and regenerating the crop for grain yield is advantageous. The ratoonability of pearl millet adds a degree of versatility that could be useful in a period of unfavourable or dry monsoon. The ratoonability saves the cost for establishing new crop and offers an opportunity of availability of green fodder at scarcity time.

Pearl millet is endowed with a rich reservior of genetic variability for various traits of yield, adaptation and quality. Exploitation of immense genetic variability in the available germplasm holds the promise of producing high yielding hybrids and open pollinated varieties adapted to a wide range of both traditional and non-traditional agro-ecological environments. The more diverse are the parents, the greater are the chances of obtaining new combinations of genes and thus more are the chances of improvement. The efficiency of a breeding method for improving a trait depends largely on its inheritance pattern (Sagar and Kapoor, 1988). Though, there are several mating designs available for evaluation of lines, the line $x$ tester design suggested by Sprague and Tatum (1942) and statistical analysis given by Kempthorne (1957) is simple and extensively used mating design for studying the combining ability of lines and genetic variances for characters.

Several other sources of male sterility inducing cytoplasms besides $\mathrm{A}_{1}\left(\mathrm{~A}_{2}, \mathrm{~A}_{3} \mathrm{~A}_{4}\right.$ and $A_{5}$ ) have been reported to diversify the cytoplasm and nuclear genetic base of pearl millet hybrids. However, commercial utilization of different CMS systems depends on several factors including their effect on agronomic traits. The iso-nuclear lines have also been established in the background of several diverse CMS sources, which provides an opportunity for studying cytoplasmic effects on the expression of different characters. Studies have indicated that cytoplasm exhibits pronounced effect on combining ability (Young and Virmani, 1990, Kumar 2002, Kumar and Sagar, 2010 and Kumar and Sagar 2015). Moreover, there are diverse opinions about combining ability effects as influenced by type of sterile cytoplasm (Yadav, 1999). Studies on combing ability involving diverse cytopasmic male sterlity system in direct sown and ratoon pearl millet crop is very scanty. Thus, a systematic and comprehensive study was carried out on combining ability for grain yield and its three 
component characters viz., 500-grain weight (g), harvest index (\%) and growth rate (g/plant/day) involving diverse CMS sources lines in direct sown and ratooned (cut regenerated) pearl millet crop.

\section{Materials and Methods}

The material for the present study consisted of six male sterile lines from five systems of male sterility namely two male sterile lines from system $\mathrm{A}_{1}\left(\mathrm{MS} 81 \mathrm{~A}_{1}\right.$, HMS $\left.8 \mathrm{~A}_{1}\right)$ and one each from $\mathrm{A}_{2}\left(\mathrm{~Pb} .313 \mathrm{~A}_{2}\right), \mathrm{A}_{3}\left(\mathrm{~Pb} .402 \mathrm{~A}_{3}\right), \mathrm{A}_{4}$

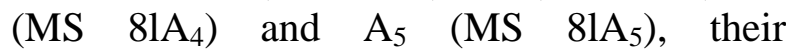
corresponding maintainer $(\mathrm{B})$ lines $\mathrm{B}_{1}(\mathrm{MS}$ 81B, HMS 8B), $\mathrm{B}_{2}$ (Pb.313B), $\mathrm{B}_{3}$ (Pb.402B), $\mathrm{B}_{4}\left(\mathrm{MS} 1 \mathrm{~B}_{4}\right)$ and $\mathrm{B}_{5}\left(\mathrm{MS}_{13} \mathrm{~B}_{5}\right)$ and twelve restorer lines viz. H90/4-5, H77/833-2, G73-107, 77/245, 77/273, CSSC 46-2, ISK 48, ICR I61, 77/180, 78/711 and Raj. 42. The six male sterile lines and their corresponding maintainer lines were crossed with twelve restorer lines in line $\mathrm{x}$ tester design. The 24 parents and 144 crosses were grown separately in contiguous blocks in randomized block design with two replications in six environments (treatments) three each viz., early sown non-cut crop $\left(E_{1}, E_{4}\right)$, ratoon crop $\left(E_{2}, E_{5}\right)$ and late sown non-ratooned $\left(E_{3}, E_{6}\right)$ at Research Farm, Bajra Section, CCS HAU, Hisar. To assess ratoon effect planting was done in four environments $E_{1}, E_{2}$ and $E_{4}, E_{5}$ earlier than normal time and crop was cut in $\mathrm{E}_{2}$ and $\mathrm{E}_{5}$ after 40 days and regenerated / ratooned. The crop in $\mathrm{E}_{3}$ and $\mathrm{E}_{6}$ was sown at time on date of cutting in $E_{2}$ and $E_{5}$, respectively. The plot size was $2 \mathrm{r} \times 2.5 \mathrm{~m} \mathrm{x}$ $0.45 \mathrm{~m}$ with $10 \mathrm{~cm}$ intra-row spacing. All the recommended agronomic practices were followed to raise a good crop. The data were recorded for grain yield and three other yield related characters viz. 500-grain weight (g), harvest index (\%) and growth rate (g /plant/day).The harvest index was calculated in percentage by formula given by Donald (1962).The growth rate was calculated as suggested by Bramel Cox et al., (1984). The analysis of variance was carried out in each of the environments according to Federer (1977). Combining ability analysis was carried out following Kempthorne (1957).

\section{Results and Discussion}

The analysis of variance for combining ability (Table 1) revealed that the mean squares due to lines, testers and lines $\mathrm{x}$ testers were highly significant when tested against the error mean sum of squares for all the characters in all the environments during both years However, the mean sum of squares due to lines and testers when tested against the interaction (lines $\times$ testes), the mean sum of squares due to lines exhibited non-significance in some environments for the traits viz. grain yield, harvest index in $\mathrm{E}_{5}$; growth rate $\left(\mathrm{E}_{2}, \mathrm{E}_{4}, \mathrm{E}_{5}\right)$, This revealed that lines did not show much variation in these environments.

The mean squares due to testers were highly significant for all the traits in all the environments except for some characters in some environments viz. grain yield and growth rate in $\mathrm{E}_{4}$ and harvest index $\left(\mathrm{E}_{6}\right)$. This revealed that testers differed more for general combining ability. Further highly significant mean squares due to lines $x$ testers suggested the hybrids differed significantly for specific combining ability for all the traits in all the environments during both the years. Further, the magnitude of mean square due to testers was invariably higher as compared to those of lines for most of the traits. This indicated that a large portion of the genetic variability in crosses was accounted by the differences in testers.

The fixed effect variances due to general combining ability (gca) and specific combining ability (sca) presented in Table 1 revealed that the magnitude of sca variances exceeded to that of gca variances for all the 
characters and also the estimates of gca and sca variances and the ratio between gca and sca variances was less than unity for all characters under study that supported for nonadditive gene action. Preponderance of nonadditive genetic control of all characters has been reported in a compendium of biometrical analysis in pearl millet by Yadav et al., (2000), Bhadalia et al., (2014), Mungra et al., (2015), Rafiq et al., (2016), Kumar et al., (2017) and Sharma et al., (2019).

The estimates of general combining ability effects of lines are important in a crop like pearl millet where large number of hybrids are developed and tested every and therefore, would help in selecting the parents for using them in hydridization. None of the lines (sterile as well as maintainer) and testers proved to be good general combiners for all the characters (Table 2) in all the environments, the crosses in general also did not express significant high sca effects in all the environments for all the characters studied. Kumar (2002), Sharma et al., (2005) and Sharma et al., (2019) also reported similar observation.

A critical examination of gca effects of lines revealed that 3 (313A) and 9 (313B) representing $\mathrm{A}^{2}$ system of male sterile cytoplasm and fertile cytoplasm combined favourably for grain yield, and growth rate. Lines 4 (402A) and 10 (402B) of $\mathrm{A}^{3}$ system proved good general combiners for 500 grain weight. The combining ability of $\mathrm{A}^{1}$ system lines 1/7 (81A/81B) and 2/8 (8A/8B) was not similar at system level. But $1(81 \mathrm{~A})$ and 7 (81B) combined better for harvest index and line 1 (81A) also combined better for grain yield. Lines $2(8 \mathrm{~A})$ and $8(8 \mathrm{~B})$ for 500 -grain weight and $8(8 \mathrm{~B})$ also combined favourably for grain yield. The lines $5\left(81 \mathrm{~A}^{4}\right)$ and 11 $\left(81 B^{4}\right)$ gave high gca effects for harvest index. The line $6\left(81 \mathrm{~A}^{5}\right)$ combined better for grain yield, and harvest index. Virk and Brar
(1993) reported that the $\mathrm{A}^{3}$ system $\left(\mathrm{Pb} 406 \mathrm{~A}^{3}\right.$ and $\mathrm{Pb} 402 \mathrm{~A}^{3}$ ) cytoplasm was better general combiner than $\mathrm{A}^{2}$ cytoplasm $\left(\mathrm{Pb}_{10} 10 \mathrm{~A}^{2}\right.$ and $\mathrm{Pb}$ $311 \mathrm{~A}^{2}$ ) for grain yield and the superiority of $\mathrm{A}^{3}$ cytoplasm for gca for plant height and ear characters. This observation is in contrast to ours. We have found high gca effects for grain yield for $\mathrm{Pb} 313 \mathrm{~A}^{2}\left(\mathrm{~A}^{2}\right)$. This is in agreement with Mangat et al., (1998) for grain yield. This is probably due to differences in maintainer nuclear background and use of different resource.

The male sterile lines and maintainers representing, different sources of cytoplasm showed substantial differences for combining ability for one or more characters (Kumar, 2002, Kumar and Sagar, 2010, Kumar and Sagar, 2015). Kumar et al., (1996) reported that none of the male sterile cytoplasmic sources in general was good combiner for all the traits studied by them. The sterile cytoplasm (A-) lines and the fertile (B-, maintainer) line expressing significant gca effects in almost similar number of environments, suggests that the cytoplasm had not the pronouncing effect on gca of a line. The effect of environment on cytoplasm was implicit from the same and differential behaviour of lines (A-line and B-line) for gca in different environments which could be due to micro climatic influences accumulated over different phenophases of plant development (Kumar, 2002).

The gca effects of near-isonuclear polycytoplasmic male sterile lines $\left(81,81 \mathrm{~A}^{4}\right.$, $81 \mathrm{~A}^{5}$ ) carrying the same nucleus of $81 \mathrm{~B}$ revealed that for grain yield significant positive effects in a number of environments though varied for the three A lines viz. 81A ( 3 environments), $81 \mathrm{~A}^{4}$ (2 environment), $81 \mathrm{~A}^{5}$ (4 environments), their $\mathrm{B}$ lines expressed significant gca effects in two environments each, but different ones. More or less similar behaviour of these lines was noted for other 
characters shows that maternal cytoplasm provided an unstable and differential substrate for the paternal genes as hypothesized by Renner and Kupper (1921) in plasmone sensitivity, this shows that same nucleus showed differential behaviour in different cytoplasm as a result of cytoplasm and nuclear (genic) interaction. Thus the expression of the phenotype is under the influence of nuclear genes, cytoplasmic factors and the interaction of two, and also certain nuclear-genotypic combinations perform better than others (Virk and Brar, 1993).

Tester 17 (77/245) emerged to be good general combiner for grain yield, harvest index and growth rate, exhibited significant positive gca effects in most of environments for these characters. Other testers showed significant positive gca effects for grain yield in most of the environments were 20 (ICR 161), 16 (CSSC46-2) and 23 (77/180). Tester 20 (ICR 161) was also good general combiner for 500 grain weight including ratoon crop. Testers 17(77/245), 13(90/4-5), 14 (H77/8332) and16 (CSSC 46-2) proved to be good general combiners for harvest index as indicated by their significant positive gca effects in three of six environments. Among testers, $23(77 / 180)$ and $17(77 / 245)$ were noted to be good combiner for growth rate in four of six environments.

A list of few crosses selected on the basis of sca for various characters and per se is given in Table 3. The estimates of sca effects for all the characters studied were inconsistent across the environments. Kumar (2002) and Sharma (2005) also reported similar observations. The cross $1 \times 14$ combined significantly higher for grain yield in all the six environments. The F1 hybrids 11 x 18 and 12 x 16 showed significant positive sca effects in most of the environments except former in $\mathrm{E}^{2}$ and $\mathrm{E}^{6}$; and latter in $\mathrm{E}^{3}$ and $\mathrm{E}^{6}$.
The crosses $1 \times 17,3$ x 24, 4 x 17, 4 x 21, 7 x $14,9 \times 19,10 \times 15$ and $11 \times 20$ expressed significant positive effects in Non-ratoon early crop $\left(\mathrm{E}^{1}, \mathrm{E}^{4}\right)$. The $\mathrm{F}_{1}$ hybrids 2 x $24,3 \mathrm{x}$ 18 and $10 \times 23$ showed their desirability in non-ratoon late crop $\left(\mathrm{E}^{3}, \mathrm{E}^{6}\right)$. The crosses $1 \mathrm{x}$ $13\left(E^{2}, E^{4}, E^{5}\right), 6 \times 13\left(E^{2}, E^{5}\right)$ and $9 \times 13\left(E^{2}\right.$, $\left.E^{3}, E^{5}\right)$ were found to be good specific combinations in ratoon crop $\left(\mathrm{E}^{2}, \mathrm{E}^{5}\right)$. The other crosses proved to be good combinations were $7 \times 21\left(E^{1}, E^{2}, E^{3}\right), 8 \times 16\left(E^{1}, E^{5}, E^{6}\right), 10$ x $16\left(E^{1}, E^{5}, E^{6}\right), 5 \times 17\left(E^{2}, E^{3}, E^{4}\right)$ and $5 x$ $24\left(E^{1}, E^{3}, E^{5}\right)$.

An appraisal of sca effects for 500-seed weight revealed that crosses $6 \times 24$ and $9 \times 15$ exhibited significant sca effects in five of six environments except former in $\mathrm{E}^{5}$ and latter in $\mathrm{E}_{1}$, respectively. The other good combinations $1 \times 16\left(E^{2}, E^{3}, E^{5}, E^{6}\right), 1 \times 21\left(E^{1}, E^{3}, E^{4}, E^{6}\right)$, $6 \times 18\left(\mathrm{E}^{2}, \mathrm{E}^{3}, \mathrm{E}^{5}, \mathrm{E}^{6}\right), 6 \times 23\left(\mathrm{E}^{2}, \mathrm{E}^{4}, \mathrm{E}^{5}, \mathrm{E}^{6}\right)$, $7 \times 21\left(E^{1}, E^{3}, E^{4}, E^{6}\right)$ and $9 \times 16\left(E^{1}, E^{3}, E^{4}\right.$, $\left.E^{6}\right)$ showed significant positive sca effects in four of the six environments. The F1 crosses 3 x 24, 4 x 21, 4 x 22, 5 x 20, 8 × 18, 8 x 23, 12 $\mathrm{x} 14$ in $\mathrm{E}^{1}$ and $\mathrm{E}^{4} ; 3 \times 15,9 \times 24,11 \times 14,11$ $\mathrm{x} 18,12 \times 18,12 \times 21$ in $\mathrm{E}^{2}$ and $\mathrm{E}^{5}$; and $1 \mathrm{x} 13$, $2 \times 24,5 \times 19,7 \times 16,8 \times 14,10 \times 15,10 \times$ 17, $11 \times 19 \mathrm{E}^{3}$ and $\mathrm{E}^{6}$ were desirable combination in non-ratoon early, ratoon and non-ratoon late sown crop, respectively.

The F1 hybrid 11 x 20 exhibited its promise with regard to sca behavior for harvest index in all environments except $\mathrm{E}^{5}$ (ratoon crop). The crosses 4 x 23, 8 x 15, 9 x 18, $10 \times 16,10$ x 23, $11 \times 17$ and $11 \times 18$ showed significant positive sca effects in four of the six environments. In both non-ratoon early sown environments $\left(E^{1}\right.$ and $\left.E^{4}\right)$ desirable specific combinations were noted to be $3 \times 24,4 \times 21$, $7 \times 20,9 \times 14,10 \times 13$ and $12 \times 16$. The F1 crosses $1 \times 24$ and $4 \times 20$ in $E^{2}$ and $E^{5}$ and crosses $2 \times 14,3 \times 20,10 \times 21$ and $10 \times 23$ in $\mathrm{E}^{3}$ and $\mathrm{E}^{6}$ showed promise for their high specific combining ability. 
Hybrids $1 \times 14\left(E^{1}, E^{4}, E^{5}, E^{6}\right), 4 \times 17\left(E^{1}, E^{3}\right.$, $\left.\mathrm{E}^{4}\right), 10 \times 15\left(\mathrm{E}^{1}, \mathrm{E}^{3}, \mathrm{E}^{4}\right)$ and $11 \times 18\left(\mathrm{E}^{1}, \mathrm{E}^{4}\right.$, $E^{5}$ ) exhibited significant positive sca effects for growth rate at least in three of the six environments. Crosses 1 x 13, 6 × 20, 9 x 13 and $10 \times 24$ showed significant positive sca effects in ratoon crop $\left(\mathrm{E}^{2}\right.$ and $\left.\mathrm{E}^{5}\right)$. Crosses $4 \mathrm{x}$ $15\left(E^{1}, E^{4}, E^{5}, E^{6}\right)$ and $7 \times 15\left(E^{1}, E^{2}, E^{3}\right)$ were found to be good combinations in different environments.

Table.1 Combining ability analysis, gca and sca variances for some quantitative characters in different environments

\begin{tabular}{|c|c|c|c|c|c|c|c|}
\hline Source of variation & d.f. & \multicolumn{6}{|c|}{ Mean sum of squares } \\
\hline \multicolumn{8}{|l|}{ Grain yield (g/plant) } \\
\hline & & E1 & E2 & E3 & E4 & E5 & E6 \\
\hline Lines & 11 & $494.60 * *$ & $321.70 * *$ & $349.93 * *$ & $373.08 * *$ & $118.90 * *+$ & $248.46 * *$ \\
\hline Testers & 11 & $206.56^{* *}$ & $176.44 * *$ & $99.91 * *$ & $113.17 * *+$ & $373.43 * *$ & $182.15 * *$ \\
\hline Lines $\times$ testers & 121 & $60.70 * *$ & $60.06 * *$ & $41.60 * *$ & $72.25 * *$ & $87.34 * *$ & $69.62 * *$ \\
\hline Error & 143 & 6.23 & $' 8.34$ & 7.23 & 9.28 & 6.30 & 7.72 \\
\hline gca variances & & 12.08 & 7.88 & 7.64 & 7.11 & 6.62 & 6.07 \\
\hline sca variances & & 27.24 & 25.84 & 17.19 & 31.50 & 40.52 & 30.95 \\
\hline gca variances/sca variances & & 0.44 & 0.30 & 0.44 & 0.23 & 0.16 & 0.20 \\
\hline \multicolumn{8}{|l|}{ 500-grain weight (g) } \\
\hline Lines & 11 & $1.90 * *$ & $2.69 * *$ & $1.05 * *$ & $1.70 * *$ & $4.47 * *$ & $2.02 * *$ \\
\hline Testers & 11 & $1.45 * *$ & $2.88 * *$ & $3.37 * *$ & $1.35 * *$ & $1.62 * *$ & $2.86 * *$ \\
\hline Lines $\times$ testers & 121 & $0.30 * *$ & $0.62 * *$ & $0.58 * *$ & $0.27 * *$ & $0.40 * *$ & $0.50 * *$ \\
\hline Error & 143 & 0.05 & 0.03 & 0.01 & 0.03 & 0.03 & 0.04 \\
\hline gca variances & & 0.06 & 0.09 & 0.07 & 0.05 & 0.11 & 0.08 \\
\hline sca variances & & 0.13 & 0.29 & 0.28 & 0.12 & 0.19 & 0.23 \\
\hline gca variances/sca variances & & 0.46 & 0.31 & 0.25 & 0.42 & 0.58 & 0.35 \\
\hline \multicolumn{8}{|l|}{ Harvest Index (\%) } \\
\hline Lines & 11 & $98.01 * *$ & $124.68 * *$ & $60.84^{* *}$ & $74.73 * *$ & $25.54 * *+$ & $125.88 * *$ \\
\hline Testers & 11 & $64.00 * *$ & $43.13 * *$ & $39.44 * *$ & $44.97 * *$ & $41.34 * *$ & $29.29 * *+$ \\
\hline Lines $\times$ testers & 121 & $13.51 * *$ & $17.22^{* *}$ & $17.75^{* *}$ & $17.47 * *$ & $18.32^{* * *}$ & $23.07 * *$ \\
\hline Error & 143 & 1.06 & 2.76 & 1.52 & 1.75 & 1.52 & 2.17 \\
\hline gca variances & & 2.81 & 2.78 & 1.34 & 1.77 & 0.63 & 2.27 \\
\hline sca variances & & 6.23 & 7.24 & 8.13 & 7.85 & 8.40 & 10.45 \\
\hline Ratio & & 0.45 & 0.38 & 0.16 & 0.23 & 0.08 & 0.22 \\
\hline \multicolumn{8}{|l|}{ Growth rate(g/plant/day) } \\
\hline Lines & 11 & $0.12 * *$ & $0.02 * *+$ & $0.26 * *$ & $0.08^{* *+}$ & $0.07 * *+$ & $0.13 * *$ \\
\hline Testers & 11 & $0.13 * *$ & $0.10 * *$ & $0.15 * *$ & $0.11^{* *+}$ & $0.26^{* *}$ & $0.16 * *$ \\
\hline Lines $\times$ testers & 121 & 0.06 & $0.03 * *$ & $0.07 * *$ & $0.06^{* *}$ & $0.040^{* * *}$ & $0.07 * *$ \\
\hline Error & 143 & 0.01 & 0.007 & 0.01 & 0.01 & 0.04 & 0.01 \\
\hline gca variances & & 0.003 & 0.001 & 0.01 & 0.002 & 0.004 & 0.003 \\
\hline sca variances & & 0.03 & 0.013 & 0.03 & 0.024 & 0.02 & 0.03 \\
\hline gca variances/sca variances & & 0.10 & 0.08 & 0.33 & 0.08 & 0.20 & 0.10 \\
\hline
\end{tabular}

*, **Significant at $\mathrm{P}=0.05$ and $\mathrm{P}=0.01$ levels, respectively.

+ Non-significant when tested against line $\mathrm{x}$ tester mean sum of squares at $\mathrm{P}=0.05$.

$E_{1}$ and $E_{1}-$ Non-ratoon early sown crop, $E_{2}$ and $E_{5}-$ Ratoon crop, $E_{3}$ and $E_{6}-$ Non-ratoon late sown crop 
Table.2 The estimates of general combining ability effects of parents (lines and testers) for different traits in six environments

\begin{tabular}{|c|c|c|c|c|c|c|c|c|c|c|c|c|c|}
\hline \multirow[t]{2}{*}{ Sr.No. } & \multirow[t]{2}{*}{ Genotype } & \multicolumn{6}{|c|}{ Grain yield(g/plant) } & \multicolumn{6}{|c|}{ 500-grain weight (g) } \\
\hline & & $\mathbf{E}_{1}$ & $\mathbf{E}_{2}$ & $\mathbf{E}_{3}$ & $\mathbf{E}_{4}$ & $\mathbf{E}_{5}$ & $\mathbf{E}_{6}$ & $\mathbf{E}_{1}$ & $\mathbf{E}_{2}$ & $\mathbf{E}_{3}$ & $\mathbf{E}_{4}$ & $\mathbf{E}_{5}$ & $\mathbf{E}_{6}$ \\
\hline & Lines & & & & & & & & & & & & \\
\hline 1 & $81 \mathrm{~A}_{1}$ & -0.71 & $1.72 *$ & 0.37 & 0.01 & $2.97 *$ & $2.29 *$ & $-0.19 *$ & $-0.25^{*}$ & $-0.12 *$ & $-0.25^{*}$ & $-0.54 *$ & $-0.33^{*}$ \\
\hline 2 & $8 \mathrm{~A}_{1}$ & $1.55^{*}$ & 1.01 & $1.65^{*}$ & 0.77 & -0.05 & $-3.10 *$ & $0.40 *$ & $0.51 *$ & $0.48^{*}$ & $0.32 *$ & $0.46^{*}$ & $0.16^{*}$ \\
\hline 3 & $\mathrm{~Pb} 313 \mathrm{~A}_{2}$ & $3.01 *$ & $2.37 *$ & $5.46^{*}$ & $1.73^{*}$ & $1.71 *$ & $-4.47 *$ & 0.06 & $-0.10 *$ & $0.12 *$ & $0.13^{*}$ & $0.16^{*}$ & 0.05 \\
\hline 4 & $\mathrm{~Pb} 402 \mathrm{~A}_{3}$ & $-8.29 *$ & $-5.63 *$ & $-6.11 *$ & $-8.72 *$ & $-3.59 *$ & $-4.08 *$ & $0.36^{*}$ & $0.37 *$ & $0.17 *$ & $0.30 *$ & $0.36^{*}$ & $0.44^{*}$ \\
\hline 5 & $81 \mathrm{~A}_{4}$ & $3.93 *$ & 1.37 & 0.38 & 1.41 & -1.26 & $3.62 *$ & $-0.21^{*}$ & $-0.33 *$ & $-0.21 *$ & $-0.15^{*}$ & $-0.34 *$ & -0.03 \\
\hline 6 & $81 \mathrm{~A}_{5}$ & $5.72 *$ & 1.45 & 0.81 & $3.14 *$ & $1.48^{*}$ & $2.16^{*}$ & $-0.27 *$ & $-0.33^{*}$ & $-0.22 *$ & $-0.36^{*}$ & $-0.48^{*}$ & -0.01 \\
\hline 7 & $81 \mathrm{~B}_{1}$ & 0.97 & 0.61 & -0.43 & $2.02 *$ & $1.56^{*}$ & 0.85 & $-0.18^{*}$ & $-0.28 *$ & $-0.36^{*}$ & $-0.27 *$ & $-0.23 *$ & $-0.37 *$ \\
\hline 8 & $8 \mathrm{~B}_{1}$ & $1.63 *$ & $2.79 *$ & 0.78 & $3.81 *$ & 0.07 & 1.18 & $0.31 *$ & $0.50^{*}$ & $0.17 *$ & $0.34 *$ & $0.41 *$ & $0.13^{*}$ \\
\hline 9 & $\mathrm{~Pb} 313 \mathrm{~B}_{2}$ & -0.94 & $1.81 *$ & $2.46^{*}$ & 0.56 & $1.74 *$ & $4.24 *$ & 0.11 & $0.13^{*}$ & $0.21 *$ & $0.11 *$ & $0.54^{*}$ & 0.10 \\
\hline 10 & $\mathrm{~Pb} 402 \mathrm{~B}_{3}$ & $-9.43^{*}$ & $-9.41^{*}$ & $-8.77 *$ & $-7.51 *$ & $-4.15^{*}$ & $-4.17 *$ & $0.26^{*}$ & $0.28 *$ & $0.14^{*}$ & $0.21 *$ & $0.43^{*}$ & $0.46^{*}$ \\
\hline 11 & $81 B_{4}$ & $1.98 *$ & 1.31 & 1.45 & $1.80^{*}$ & $-1.51 *$ & -1.02 & $-0.28 *$ & $-0.25^{*}$ & -0.04 & -0.05 & $-0.24 *$ & $-0.17 *$ \\
\hline \multirow[t]{3}{*}{12} & $81 B_{5}$ & 0.59 & 0.60 & $1.94 *$ & 0.97 & 1.04 & $2.48 *$ & $-0.36^{*}$ & $-0.25^{*}$ & $-0.35^{*}$ & $-0.32^{*}$ & $-0.53 *$ & $-0.44 *$ \\
\hline & S.E.(d) & 0.72 & 0.83 & 0.78 & 0.88 & 0.72 & 0.80 & 0.06 & 0.05 & 0.03 & 0.05 & 0.05 & 0.06 \\
\hline & Testers & & & & & & & & & & & & \\
\hline 13 & $\mathrm{H} 90 / 4-5$ & -1.16 & $1.93 *$ & -1.34 & -0.79 & $-4.54 *$ & -1.05 & -0.04 & $-0.19 *$ & $-0.22 *$ & 0.04 & -0.01 & $-0.22 *$ \\
\hline 14 & $\mathrm{H} 77 / 833-2$ & $-2.29 *$ & $3.05 *$ & -0.11 & -1.67 & $-3.80 *$ & $-1.93^{*}$ & $-0.21^{*}$ & $-0.46^{*}$ & $-0.29 *$ & $-0.18^{*}$ & $-0.20^{*}$ & $-0.34 *$ \\
\hline 15 & G73-107 & -0.48 & 0.94 & $-1.67 *$ & 1.07 & $-2.97 *$ & $-3.15^{*}$ & $-0.24 *$ & $0.12 *$ & $-0.06 *$ & $-0.17 *$ & 0.02 & $-0.34 *$ \\
\hline 16 & CSSC46-2 & $-3.62 *$ & $3.77 *$ & $-1.63 *$ & $-1.76^{*}$ & $4.41 *$ & $4.74^{*}$ & $0.33^{*}$ & $0.17 *$ & $-0.29 *$ & $0.14^{*}$ & $0.14^{*}$ & -0.06 \\
\hline 17 & $77 / 245$ & $5.29 *$ & $-4.45^{*}$ & $5.00 *$ & $2.55^{*}$ & $2.30 *$ & $1.85^{*}$ & $-0.27 *$ & $-0.42 *$ & $-0.10^{*}$ & $-0.31 *$ & $-0.34^{*}$ & 0.05 \\
\hline 18 & $78 / 711$ & 0.66 & 1.23 & -0.81 & 0.41 & 0.28 & $1.80 *$ & $0.39 *$ & $0.41 *$ & $0.11 *$ & $0.37 *$ & $0.20 *$ & $0.42 *$ \\
\hline 19 & $77 / 273$ & -0.45 & 1.20 & -0.53 & -0.04 & $3.47 *$ & 0.13 & $-0.15^{*}$ & $-0.28 *$ & $0.07 *$ & $-0.10^{*}$ & $-0.19 *$ & -0.03 \\
\hline 20 & ICR 161 & $3.67 *$ & $-4.25^{*}$ & 0.86 & $2.26^{*}$ & $5.10^{*}$ & $2.28^{*}$ & 0.07 & $0.29 *$ & $0.60 *$ & 0.09 & $0.51 *$ & $0.63^{*}$ \\
\hline 21 & ISK-48 & 0.02 & -0.35 & $1.80 *$ & -0.40 & $3.55^{*}$ & $-2.24 *$ & $0.40^{*}$ & $0.32 *$ & $0.73^{*}$ & $0.49 *$ & $0.32 *$ & $0.45^{*}$ \\
\hline 22 & $77 / 28-2$ & -0.17 & -0.98 & $-2.60 *$ & 0.31 & $1.80 *$ & -0.02 & $-0.12 *$ & $0.40^{*}$ & $0.28 *$ & -0.03 & $-0.11^{*}$ & -0.03 \\
\hline 23 & $77 / 180$ & $3.23 *$ & 1.03 & -0.47 & $2.93^{*}$ & $-3.76^{*}$ & $2.43^{*}$ & $-0.18^{*}$ & $-0.48^{*}$ & $-0.41^{*}$ & $-0.19 *$ & $-0.34^{*}$ & $-0.50 *$ \\
\hline \multirow[t]{2}{*}{24} & Raj-42 & $-4.71 *$ & $-3.13 *$ & 1.28 & $-4.86^{*}$ & $-5.85 *$ & $-4.84^{*}$ & 0.02 & $0.13^{*}$ & $-0.40^{*}$ & $-0.14 *$ & 0.01 & -0.03 \\
\hline & SE.(d) & 0.72 & 0.83 & 0.78 & 0.88 & 0.72 & 0.80 & 0.06 & 0.05 & 0.03 & 0.05 & 0.05 & 0.06 \\
\hline
\end{tabular}

*Significant at $\mathrm{P}=0.05$ level.

$\mathrm{E}_{1}$ and $\mathrm{E}_{1}-$ Non-ratoon early sown crop, $\mathrm{E}_{2}$ and $\mathrm{E}_{5}-\mathrm{Ratoon}$ crop, $\mathrm{E}_{3}$ and $\mathrm{E}_{6}-$ Non-ratoon late sown crop 
Table.2 Continued

\begin{tabular}{|c|c|c|c|c|c|c|c|c|c|c|c|c|c|}
\hline \multirow{2}{*}{$\begin{array}{l}\text { Sr. } \\
\text { No. }\end{array}$} & \multirow[t]{2}{*}{ Genotype } & \multicolumn{6}{|c|}{ Harvest Index (\%) } & \multicolumn{6}{|c|}{ Growth rate (g/plant/day) } \\
\hline & & $\mathbf{E}_{1}$ & $\mathbf{E}_{2}$ & $\mathbf{E}_{3}$ & $\mathbf{E}_{4}$ & $\mathbf{E}_{5}$ & $\mathbf{E}_{6}$ & $\mathbf{E}_{1}$ & $\mathbf{E}_{2}$ & $\mathbf{E}_{3}$ & $\mathbf{E}_{4}$ & $\mathbf{E}_{5}$ & $\mathbf{E}_{6}$ \\
\hline & \multicolumn{13}{|l|}{ Lines } \\
\hline 1 & $81 \mathrm{~A}_{1}$ & $0.70 *$ & $1.77 *$ & $1.65^{*}$ & 0.57 & 0.26 & $1.44^{*}$ & -0.05 & -0.03 & $-0.07 *$ & -0.01 & $0.08 *$ & 0.02 \\
\hline 2 & $8 \mathrm{~A}_{1}$ & $1.40 *$ & 0.52 & 0.03 & $0.93 *$ & $\cdot 0.32$ & $-1.04 *$ & -0.02 & 0.02 & $0.07 *$ & -0.01 & 0.02 & $-0.09 *$ \\
\hline 3 & $\mathrm{~Pb} 313 \mathrm{~A}_{2}$ & 0.27 & 0.66 & $1.64 *$ & -0.23 & $1.10 *$ & $-1.22 *$ & $012 *$ & $0.12 *$ & $0.15^{*}$ & $0.08 *$ & 0.02 & $-0.13 *$ \\
\hline 4 & $\mathrm{~Pb} 402 \mathrm{~A}_{3}$ & $-3.83 *$ & $-2.76^{*}$ & $-3.35 *$ & $-3.92 *$ & -0.37 & $-3.60 *$ & $-0.09 *$ & $-0.04 *$ & 0.05 & $-0.11 *$ & $-0.10 *$ & $0.08 *$ \\
\hline 5 & $81 \mathrm{~A}_{4}$ & $1.37 *$ & $0.95 *$ & 0.55 & $0.91 *$ & 0.03 & $2.97 *$ & $0.08 *$ & 0.02 & -0.01 & 0.00 & $-0.04 *$ & -0.02 \\
\hline 6 & $81 \mathrm{~A}_{5}$ & $2.59 *$ & $1.36^{*}$ & $1.4 \mathrm{~B}^{*}$ & $1 . \mathrm{B} 2 *$ & 0.70 & $2.01 *$ & 0.03 & -0.02 & $-0.06^{*}$ & 0.00 & 0.03 & -0.02 \\
\hline 7 & $81 B_{1}$ & 0.49 & $1.14 *$ & 0.39 & $1.45^{*}$ & -0.12 & -0.60 & 0.01 & -0.02 & -0.05 & 0.01 & $0.06 *$ & 0.05 \\
\hline 8 & $8 \mathrm{~B}_{1}$ & $0.99 *$ & $1.21 *$ & 0.25 & $1.11 *$ & 0.64 & -0.60 & 0.00 & 0.03 & 0.04 & 0.05 & -0.02 & $0.06^{*}$ \\
\hline 9 & $\mathrm{~Pb} 313 \mathrm{~B}_{2}$ & $-1.26 *$ & -0.12 & 0.17 & -0.45 & -0.64 & $1.87 *$ & $0.07 *$ & $0.04 *$ & $0.12-$ & 0.04 & $0.07 *$ & $0.09 *$ \\
\hline 10 & $\mathrm{~Pb}_{402 \mathrm{~B}_{3}}$ & $-3.93 *$ & $6.24^{*}$ & $-2.94 *$ & $-304 "$ & $-2.23 *$ & $-4.15^{*}$ & $-0.13 *$ & -0.02 & $-0.24 *$ & $-0.11 *$ & $-0.05 *$ & $0.09 *$ \\
\hline 11 & $81 B_{4}$ & $0.54 *$ & $0.89 *$ & 0.04 & 0.14 & $-0.80^{*}$ & 0.61 & 0.01 & 0.02 & 4.00 & 0.04 & -0.03 & $-0.08 *$ \\
\hline \multirow[t]{3}{*}{12} & $81 B_{5}$ & 0.55 & 0.61 & 0.10 & 0.70 & $1.81 *$ & $2.30 *$ & -0.01 & -0.01 & $0.05^{*}$ & 0.02 & $-0.04 *$ & -0.05 \\
\hline & S.E.(d) & 0.30 & 0.48 & 0.36 & 0.38 & 0.36 & 0.43 & 0.03 & 0.02 & 0.03 & 0.03 & 0.02 & 0.03 \\
\hline & \multicolumn{13}{|c|}{ Testers } \\
\hline 13 & $\mathrm{H} 90 / 4-5$ & $1.03 *$ & 0.88 & -0.23 & $1.14 *$ & $-1.63^{*}$ & $1.30 *$ & $-0.11 *$ & $0.04 *$ & $-0.07 *$ & $-0.07 *$ & $-0.08 *$ & $-0.06^{*}$ \\
\hline 14 & H77/833-2 & $0.74 *$ & $1.04 *$ & $-0.87^{*}$ & $0.96^{*}$ & 0.06 & 0.03 & $-0.07 *$ & $0.10^{*}$ & $0.13^{*}$ & -0.05 & $-0.10 *$ & 0.04 \\
\hline 15 & G73-107 & -0.31 & $1.21 *$ & $-0.95^{*}$ & $1.00 *$ & 0.45 & $-116^{*}$ & 0.00 & $-0.04 *$ & -0.03 & -0.03 & $-0.09 *$ & $-0.09 *$ \\
\hline 16 & CSSC46-2 & $-1.45^{*}$ & $1.89 *$ & $1.27 *$ & -0.50 & $0.75^{*}$ & 0.65 & $-0.10 *$ & 0.01 & $-0.16^{*}$ & $-0.07 *$ & $0.09 *$ & $0.13^{*}$ \\
\hline 17 & $77 / 245$ & $1.81 *$ & -0.40 & $2.10 *$ & $0.99 *$ & 0.70 & 0.28 & $0.14 *$ & $-0.09 *$ & $0.13 *$ & 0.05 & $0.06 *$ & $0.08 *$ \\
\hline 18 & $78 / 711$ & -0.48 & 0.36 & 0.19 & -0.25 & $-139 *$ & $-1.06 *$ & 0.03 & 0.01 & -0.03 & 0.05 & $0.04 *$ & $0.06^{*}$ \\
\hline 19 & $77 / 273$ & 0.10 & -0.36 & $-1.02 *$ & 0.19 & $1.38 *$ & $-1.10 *$ & -0.02 & $0.06^{*}$ & 0.02 & $-0.06 *$ & $0.05 *$ & 0.01 \\
\hline 20 & ICR $\cdot 161$ & $0.74 *$ & $-1.77^{*}$ & 0.51 & -0.21 & $0.72 *$ & 0.54 & 0.02 & $-0.10 *$ & -0.01 & 0.05 & $0.12 *$ & -0.02 \\
\hline 21 & ISK-48 & $1.62 *$ & 0.93 & $1.62 *$ & 0.71 & $0.95 *$ & 0.55 & -0.04 & $-0.05^{*}$ & 0.04 & -0.04 & $0.08 *$ & $-0.09 *$ \\
\hline 22 & $77 / 28-2$ & $-0.67 *$ & -0.52 & $-2.52^{*}$ & -0.38 & -0.01 & $1.56^{*}$ & 0.00 & $-0.04 *$ & -0.03 & 0.02 & $0.04 *$ & $-0.09 *$ \\
\hline 23 & $77 / 180$ & $1.04 *$ & -0.41 & 0.06 & 0.26 & $0.98 *$ & 0.49 & $0.07 *$ & $0.07 *$ & 0.00 & $0.15^{*}$ & $-0.14 *$ & $0.11^{*}$ \\
\hline 24 & Raj-42 & $-4.18 *$ & $-2.86^{*}$ & -0.16 & $-3.91 *$ & $-2.97 *$ & $2.08 *$ & $0.06^{*}$ & 0.03 & 0.04 & 0.00 & $-0.09 *$ & $-0.09 *$ \\
\hline \multicolumn{2}{|c|}{ S.E.(d) } & 0.3 & 0.48 & 0.36 & 0.38 & 0.36 & 0.43 & 0.03 & 0.02 & 0.03 & 0.03 & 0.02 & 0.03 \\
\hline
\end{tabular}

*Significant at $\mathrm{P}=0.05$ level.

$\mathrm{E}_{1}$ and $\mathrm{E}_{1}-$ Non-ratoon early sown crop, $\mathrm{E}_{2}$ and $\mathrm{E}_{5}-$ Ratoon crop, $\mathrm{E}_{3}$ and $\mathrm{E}_{6}-\mathrm{Non}$-ratoon late sown crop 
Table.3 Specific combining ability effects and per se performance (in parentheses) for top fourteen $\mathrm{F}_{1}$ hybrids for some quantitative characters in different environments

\begin{tabular}{|c|c|c|c|c|c|c|c|c|c|c|c|c|c|c|c|}
\hline \multirow[t]{2}{*}{$F_{1}$ Hybrids } & \multicolumn{7}{|c|}{ Grain yield (g/plant) } & \multirow{2}{*}{ F 1 Hybrids } & \multicolumn{7}{|c|}{ 500-grain weight (g) } \\
\hline & $\mathbf{E}_{1}$ & $\mathbf{E}_{2}$ & $\mathbf{E}_{3}$ & $\mathbf{E}_{4}$ & $\mathbf{E}_{5}$ & $\mathbf{E}_{6}$ & $\begin{array}{c}\text { Environ- } \\
\text { mental mean }\end{array}$ & & $\mathbf{E}_{1}$ & $\mathbf{E}_{2}$ & $\mathbf{E}_{3}$ & $\mathbf{E}_{4}$ & $\mathbf{E}_{5}$ & $\mathbf{E}_{6}$ & $\begin{array}{c}\text { Environ- } \\
\text { mental mean }\end{array}$ \\
\hline \multirow[t]{2}{*}{$1 \times 14$} & $5.70^{*}$ & $5.90 *$ & $7.90 *$ & $9.72 *$ & $7.45^{*}$ & $6.14^{*}$ & & $9 \times 15$ & 0.36 & $1.40 *$ & $0.35 *$ & $0.38 *$ & $0.90 *$ & $0.44 *$ & \\
\hline & $(35.8)$ & $(38.8)$ & $(33.6)$ & $(39.9)$ & $(32.8)$ & $(33.1)$ & $(35.7)$ & & $(4.7)$ & $(5.8)$ & $(5.1)$ & $(4.7)$ & $(5.6)$ & $(4.6)$ & $(5.1)$ \\
\hline \multirow[t]{2}{*}{$11 \times 18$} & $13.06 *$ & -0.57 & $5.84^{*}$ & $11.06 *$ & $9.65^{*}$ & $-5.88 *$ & & $6 \times 24$ & $0.47 *$ & $0.34 *$ & $0.85^{*}$ & $0.39 *$ & $-0.62 *$ & $0.86^{*}$ & \\
\hline & $(48.8)$ & $(30.1)$ & $(31.7)$ & $(45.1)$ & $(34.6)$ & $(21.5)$ & $(35.3)$ & & $(4.7)$ & $(4.3)$ & $(4.8)$ & $(4.3)$ & $(3.0)$ & $(5.2)$ & $(4.4)$ \\
\hline \multirow[t]{2}{*}{$12 \times 16$} & $7.53 *$ & $5.91 *$ & 5.18 & $6.05 *$ & $9.47 *$ & $-13.83^{*}$ & & $1 \times 16$ & 0.05 & $0.51 *$ & $0.55^{*}$ & -0.23 & $0.73 *$ & $0.48 *$ & \\
\hline & (37.6) & $(38.4)$ & $(30.7)$ & $(37.1)$ & (41.1) & $(20.0)$ & $(34.2)$ & & $(4.6)$ & $(4.6)$ & $(4.7)$ & $(4.1)$ & $(4.4)$ & $(4.5)$ & $(4.5)$ \\
\hline \multirow[t]{2}{*}{$3 \times 18$} & $9.23 *$ & 0.27 & $6.53^{*}$ & 5.53 & $-5.27 *$ & $15.57 *$ & & $2 \times 20$ & -0.36 & $0.35^{*}$ & $0.38 *$ & -0.25 & $0.55^{*}$ & $0.72 *$ & \\
\hline & $(46.0)$ & $(32.0)$ & $(36.4)$ & $(39.5)$ & (22.9) & $(39.5)$ & $(36.1)$ & & $(4.6)$ & $(5.3)$ & (6.1) & $(4.6)$ & $(5.6)$ & (5.9) & $(5.3)$ \\
\hline \multirow[t]{2}{*}{$1 \times 13$} & 3.27 & $7.72 *$ & 2.85 & $6.04 *$ & $8.59 *$ & 2.26 & & $6 \times 18$ & 0.05 & $0.47 *$ & $0.78^{*}$ & -0.27 & $0.37 *$ & $0.92 *$ & \\
\hline & $(34.5)$ & $(39.5)$ & $(27.1)$ & (37.1) & (33.2) & $(30.1)$ & (33.6) & & (4.6) & $(4.7)$ & $(5.3)$ & (4.1) & $(4.2)$ & $(5.7)$ & $(48)$ \\
\hline \multirow[t]{2}{*}{$2 \times 24$} & $12.56^{*}$ & -1.31 & $9.45^{*}$ & 3.85 & 1.53 & $6.34 *$ & & $6 \times 23$ & 0.15 & $1.03 *$ & -0.14 & $0.52 *$ & 0.63 & $0.41 *$ & \\
\hline & $(42.5)$ & (24.7) & $(37.6)$ & (31.6) & $(21.8)$ & $(25.0)$ & $(35.5)$ & & $(4.1)$ & $(4.4)$ & $(3.8)$ & $(4.4)$ & (3.9) & $(4.3)$ & (4.1) \\
\hline \multirow[t]{2}{*}{$4 \times 21$} & $6.07 *$ & 4.45 & 1.29 & $7.39 *$ & $\left(5.35^{*}\right.$ & 3.58 & & $7 \times 21$ & $0.84 *$ & 0.17 & $0.56^{*}$ & $1.05^{*}$ & 0.06 & $0.59 *$ & \\
\hline & $(30.9)$ & (26.6) & $(22.2)$ & $(30.1)$ & $(31.5)$ & (16.7) & (26.3) & & $(5.5)$ & $(4.4)$ & $(5.5)$ & $(5.7)$ & $(4.2)$ & $(5.1)$ & $(5.1)$ \\
\hline \multirow[t]{2}{*}{$5 \times 17$} & 2.18 & $8.45^{*}$ & $9.40 *$ & $6.81 *$ & 1.38 & -3.97 & & $1 \times 13$ & 0.21 & -0.18 & $0.23^{*}$ & 0.05 & $0.70 *$ & $0.40^{*}$ & \\
\hline & $(44.5)$ & $(33.5)$ & $(40.0)$ & $(42.6)$ & (28.6) & $(28.1)$ & $(36.2)$ & & $(4.4)$ & $(3.5)$ & $(4.5)$ & $(4.2)$ & $(4.2)$ & $(4.2)$ & (4 2) \\
\hline \multirow[t]{2}{*}{$7 \times 21$} & $7.71 *$ & $6.11 *$ & $8.22 *$ & 0.24 & 3.50 & 2.79 & & $3 \times 15$ & 0.17 & $1.42 *$ & $1.16^{*}$ & 0.20 & $0.52 *$ & 0.19 & \\
\hline & $(41.8)$ & $(34.5)$ & $(34.8)$ & (33.7) & $(34.8)$ & $(28.0)$ & (34.6) & & $(4.4)$ & $(5.6)$ & $(5.8)$ & (4.6) & $(4.8)$ & (4.3) & (4.9) \\
\hline \multirow[t]{2}{*}{$8 \times 16$} & $6.88^{*}$ & -2.48 & 0.83 & 3.71 & $6.44 *$ & $5.48^{*}$ & & $4 \times 22$ & $0.70 *$ & -0.21 & $0.22 *$ & $0.53 *$ & 0.16 & 0.35 & \\
\hline & $(38.0)$ & $(32.2)$ & $(25.2)$ & (37.6) & $(37.1)$ & $(38.0)$ & $(34.7)$ & & $(5.4)$ & $(4.7)$ & $(5.3)$ & $(5.2)$ & $(4.5)$ & $(5.2)$ & $(5.0)$ \\
\hline \multirow[t]{2}{*}{$9 \times 13$} & 1.00 & $7.93^{*}$ & $6.97 *$ & -1.11 & $7.62 *$ & 2.21 & & $4 \times 21$ & $0.63 *$ & $-0.81 *$ & -0.15 & $0.45^{*}$ & $0.50 *$ & -0.34 & \\
\hline & $(32.0)$ & $(39.8)$ & (33.3) & $(30.5)$ & $(31.0)$ & $(32.0)$ & (33.1) & & $(5.8)$ & $(4.0)$ & $(5.3)$ & $(5.6)$ & $(5.3)$ & $(4.9)$ & $(5.2)$ \\
\hline \multirow[t]{2}{*}{$10 \times 16$} & $5.75^{*}$ & 0.42 & -0.12 & 2.74 & $11.86^{*}$ & $7.32 *$ & & $9 \times 19$ & $0.66^{*}$ & $1.69 *$ & $0.20 *$ & -0.08 & 0.12 & -0.15 & \\
\hline & $(25.8)$ & (22.9) & (14.7) & (25.3) & $(38.3)$ & (34.5) & (26.9) & & $(5 . \mathrm{I})$ & $(5.7)$ & $(5.1)$ & $(4.3)$ & $(4.6)$ & $(4.3)$ & $(4.8)$ \\
\hline \multirow[t]{2}{*}{$11 \times 20$} & $5.34 *$ & 2.42 & 4.58 & $10.11 *$ & -3.27 & $8.93 *$ & & 10x 17 & 0.30 & $0.58 *$ & $0.34 *$ & 0.20 & -0.11 & $0.72 *$ & \\
\hline & $(44.1)$ & (27.6) & $(32.1)$ & $(46.0)$ & $(26.5)$ & $(36.8)$ & $(35.5)$ & & $(4.7)$ & $(4.6)$ & $(5.0)$ & $(4.5)$ & (4.10) & $(5.6)$ & (4.7) \\
\hline \multirow[t]{2}{*}{$9 \times 15$} & $5.32 *$ & $7.63^{*}$ & -1.71 & $7.63 *$ & $-9.45^{*}$ & 0.31 & & $11 \times 19$ & -0.29 & $-0.57 *$ & $1.37^{*}$ & $0.85^{*}$ & -0.08 & $1.68 *$ & \\
\hline & $(37.0)$ & $(38.5)$ & $(24.3)$ & (41.1) & (15.5) & $(28.0)$ & (30.7) & & $(3.7)$ & $(3.1)$ & $(6.0)$ & $(5.1)$ & (3.6) & $(5.9)$ & (4.6) \\
\hline S.E.(Sij) & 2.50 & 2.89 & 2.69 & 3.05 & 2.51 & 2.78 & & S.E.(Sij) & 0.22 & 0.17 & 0.10 & 0.17 & 0.17 & 0.2 & \\
\hline
\end{tabular}

*Significant at $\mathrm{P}=0.05$ level.

$\mathrm{E}_{1}$ and $\mathrm{E}_{4}-$ Non-ratoon early sown crop, $\mathrm{E}_{2}$ and $\mathrm{E}_{5}-$ Ratoon crop, $\mathrm{E}_{3}$ and $\mathrm{E}_{6}-$ Non-ratoon late sown crop 
Table.3 Continued

\begin{tabular}{|c|c|c|c|c|c|c|c|c|c|c|c|c|c|c|c|}
\hline \multirow{2}{*}{$\begin{array}{c}F_{1} \\
\text { Hybrids }\end{array}$} & \multicolumn{7}{|c|}{ Growth rate (g/plant/day } & \multirow{2}{*}{$\begin{array}{c}F_{1} \\
\text { Hybrids }\end{array}$} & \multicolumn{7}{|c|}{ Harvest index (\%) } \\
\hline & $\mathbf{E}_{1}$ & $\mathbf{E}_{2}$ & $\mathbf{E}_{3}$ & $\mathbf{E}_{4}$ & $\mathbf{E}_{5}$ & $\mathbf{E}_{6}$ & $\begin{array}{c}\text { Environ- } \\
\text { mental } \\
\text { mean }\end{array}$ & & $\mathbf{E}_{1}$ & $\mathbf{E}_{2}$ & $\mathbf{E}_{3}$ & $\mathbf{E}_{4}$ & $\mathbf{E}_{5}$ & $\mathbf{E}_{6}$ & $\begin{array}{c}\text { Environ- } \\
\text { mental } \\
\text { mean }\end{array}$ \\
\hline \multirow[t]{2}{*}{$1 \times 14$} & $\begin{array}{c}0.20 \\
*\end{array}$ & 0.08 & 0.18 & $\begin{array}{c}0.34 \\
*\end{array}$ & $0.14 *$ & $\begin{array}{c}0.30 \\
*\end{array}$ & & $11 \times 20$ & $2.19 *$ & $4.41 *$ & $2.49 *$ & $5.15^{*}$ & 1.94 & $3.00 *$ & \\
\hline & $(1.4)$ & (1.1) & (1.4) & $(1.4)$ & $(0.9$ & $(1.5)$ & (1.3) & & $\begin{array}{c}(32.3 \\
)\end{array}$ & (30) & $\begin{array}{c}(30.7 \\
)\end{array}$ & $\begin{array}{c}(33.7 \\
)\end{array}$ & $\begin{array}{c}(30.9 \\
)\end{array}$ & (31.9) & (31.6) \\
\hline \multirow[t]{2}{*}{$4 \times 15$} & $\begin{array}{c}0.32 \\
*\end{array}$ & -0.07 & 0.13 & $\begin{array}{c}0.36 \\
*\end{array}$ & $0.19 *$ & $\begin{array}{c}0.33 \\
*\end{array}$ & & $4 \times 23$ & $5.39 *$ & 0.05 & $6.58 *$ & $7.03 *$ & 1.17 & $7.78 *$ & \\
\hline & $(1.6)$ & $(0.8)$ & (1.2) & $(1.4)$ & $(0.7)$ & (1.5) & $(1.2)$ & & $\begin{array}{c}(31.3 \\
)\end{array}$ & (23.3) & (31) & $\begin{array}{c}(32.4 \\
)\end{array}$ & $\begin{array}{c}(30.9 \\
)\end{array}$ & $(32.4)$ & $(30.1)$ \\
\hline \multirow[t]{2}{*}{$1 \times 13$} & $\begin{array}{c}0.27 \\
*\end{array}$ & $\begin{array}{c}0.17 \\
*\end{array}$ & 0.09 & 0.19 & $0.22 *$ & 0.18 & & $8 \times 15$ & $3.53 *$ & -1.73 & $5.33 *$ & $2.83 *$ & -0.11 & $4.47 *$ & \\
\hline & $(1.4)$ & $(1.1)$ & (1.1) & $(1.2)$ & $(1.0)$ & (1.3) & (1.1) & & $\begin{array}{c}(32.9 \\
)\end{array}$ & $(27.2)$ & $\begin{array}{c}(32.3 \\
)\end{array}$ & $\begin{array}{c}(33.6 \\
)\end{array}$ & $\begin{array}{c}(30.1 \\
)\end{array}$ & $(30.4)$ & (31.1) \\
\hline \multirow[t]{2}{*}{$7 \times 15$} & $\begin{array}{c}0.23 \\
*\end{array}$ & $\begin{array}{c}0.17 \\
*\end{array}$ & $\begin{array}{c}0.25 \\
*\end{array}$ & 0.14 & 0.00 & -0.16 & & $9 \times 18$ & $2.10^{*}$ & $3.78^{*}$ & -0.15 & $2.97 *$ & $3.21 *$ & 0.62 & \\
\hline & $(1.6)$ & $(1.0)$ & $(1.3)$ & (1.3) & $(0.7)$ & (1.0) & (1.1) & & $\begin{array}{c}29.1 \\
)\end{array}$ & $(30.5)$ & $\begin{array}{c}(27.9 \\
)\end{array}$ & $\begin{array}{c}(30.9 \\
)\end{array}$ & $\begin{array}{c}(30.3 \\
)\end{array}$ & $(29.2)$ & $(29.6)$ \\
\hline \multirow[t]{2}{*}{$9 \times 22$} & 0.14 & 0.14 & $\begin{array}{c}0.34 \\
*\end{array}$ & $\begin{array}{c}0.20 \\
*\end{array}$ & $0.23 *$ & 0.11 & & $10 \times 16$ & $3.80 *$ & 1.70 & 0.88 & $4.89 *$ & $5.76^{*}$ & $3.05^{*}$ & \\
\hline & (1.6) & (1.1) & $(1.6)$ & $(1.4)$ & (1.1) & (1.3) & (1.3) & & $\begin{array}{c}(27.1 \\
)\end{array}$ & $(23.8)$ & $\begin{array}{c}(26.9 \\
)\end{array}$ & (30) & $\begin{array}{c}(33.4 \\
)\end{array}$ & (27.3) & $(28.1)$ \\
\hline \multirow[t]{2}{*}{$10 \times 15$} & $\begin{array}{c}0.40 \\
*\end{array}$ & 0.03 & $\begin{array}{c}0.21 \\
*\end{array}$ & $\begin{array}{c}0.26 \\
*\end{array}$ & -0.03 & 0.03 & & $10 \times 23$ & $2.46^{*}$ & 1.85 & $4.38 *$ & 2.43 & $4.23 *$ & $6.27 *$ & \\
\hline & $(1.6)$ & $(0.9)$ & $(1.1)$ & $(1.3)$ & $(0.6)$ & (1.2) & (1.1) & & $\begin{array}{c}(28.3 \\
)\end{array}$ & $(21.7)$ & $\begin{array}{c}(29.2 \\
)\end{array}$ & $\begin{array}{c}(28.3 \\
)\end{array}$ & $\begin{array}{c}(32.1 \\
)\end{array}$ & $(30.3)$ & $(28.3)$ \\
\hline \multirow[t]{2}{*}{$11 \times 18$} & $\begin{array}{c}0.31 \\
*\end{array}$ & 0.15 & 0.07 & $\begin{array}{c}0.22 \\
*\end{array}$ & $0.16^{*}$ & -0.23 & & $11 \times 17$ & $2.26^{*}$ & -1.89 & -0.21 & $3.15^{*}$ & $3.64 *$ & $3.59 *$ & \\
\hline & $(1.7)$ & (1.1) & $(1.3$ & $(1.5$ & $(0.9)$ & $(0.9)$ & $(1.2$ & & $\begin{array}{c}(33.4 \\
)\end{array}$ & $(25.1)$ & $\begin{array}{c}(29.6 \\
)\end{array}$ & $\begin{array}{c}(32.9 \\
)\end{array}$ & $\begin{array}{c}(32.6 \\
)\end{array}$ & $(32.2)$ & $(31.0)$ \\
\hline \multirow[t]{2}{*}{$4 \times 17$} & $\begin{array}{c}0.25 \\
*\end{array}$ & -0.01 & $\begin{array}{c}0.37 \\
*\end{array}$ & $\begin{array}{c}0.30 \\
*\end{array}$ & $0.12 *$ & 0.07 & & $11 \times 18$ & $2.81 *$ & $3.28^{*}$ & $2.72 *$ & $2.89 *$ & $4.14 *$ & 0.13 & \\
\hline & (1.6) & $(0.8)$ & (1.6) & (1.4) & $(0.6)$ & (1.4) & (1.2) & & (31.7 & $(24.4)$ & (30.7 & (31.4 & (31.0 & (27.4) & (29.4) \\
\hline
\end{tabular}




\section{Int.J.Curr.Microbiol.App.Sci (2020) 9(2): 486-499}

\begin{tabular}{|c|c|c|c|c|c|c|c|c|c|c|c|c|c|c|c|}
\hline & & & & & & & & & ) & & ) & ) & ) & & \\
\hline \multirow[t]{2}{*}{$2 \times 24$} & 0.09 & 0.08 & 0.08 & $\begin{array}{c}0.20 \\
*\end{array}$ & $0.18 *$ & 0.2 & & $1 \times 24$ & -1.03 & $3.50 *$ & -1.67 & $3.30 *$ & $5.81 *$ & -0.18 & \\
\hline & (1.5) & (1.1) & (1.4) & (1.3) & $(0.9)$ & (1.2) & (1.2) & & $\begin{array}{c}(24.2 \\
)\end{array}$ & (28.9) & $\begin{array}{c}(27.5 \\
)\end{array}$ & $\begin{array}{c}(28.6 \\
)\end{array}$ & $\begin{array}{c}(32.6 \\
)\end{array}$ & $(26.9)$ & $(28.0)$ \\
\hline \multirow[t]{2}{*}{$3 \times 18$} & 0.17 & 0.01 & $\begin{array}{c}0.45 \\
*\end{array}$ & 0.04 & 0.10 & $\begin{array}{c}0.35 \\
*\end{array}$ & & $2 \times 14$ & $2.04 *$ & -0.64 & $3.56^{*}$ & -0.06 & -1.25 & $3.99 *$ & \\
\hline & (1.7) & $(1.0)$ & (1.7) & (1.3) & $(0.9)$ & (1.4) & (1.3) & & $\begin{array}{c}(32.9 \\
)\end{array}$ & $(27.4)$ & $\begin{array}{c}(30.4 \\
)\end{array}$ & $\begin{array}{c}(30.5 \\
)\end{array}$ & $\begin{array}{c}(27.6 \\
)\end{array}$ & $(30.7)$ & (29.9) \\
\hline \multirow[t]{2}{*}{$6 \times 20$} & 0.04 & $\begin{array}{c}0.21 \\
*\end{array}$ & 0.17 & 0.1 & $0.13 *$ & 0.16 & & $4 \times 21$ & $3.08 *$ & 0.04 & -1.13 & $5.00 *$ & $4.81 *$ & 1.56 & \\
\hline & (1.4) & $(1.0)$ & (1.3) & (1.3) & $(1.0)$ & (1.3) & $(1.2)$ & & $\begin{array}{c}29.6 \\
)\end{array}$ & $(24.7)$ & $\begin{array}{c}24.8 \\
)\end{array}$ & $\begin{array}{c}(30.4 \\
)\end{array}$ & $\begin{array}{c}(34.5 \\
)\end{array}$ & $(26.2)$ & $(28.4)$ \\
\hline \multirow[t]{2}{*}{$4 \times 19$} & $\begin{array}{c}0.30 \\
*\end{array}$ & 0.06 & 0.1 & 0.19 & -0.01 & $\begin{array}{c}0.24 \\
*\end{array}$ & & $11 \times 19$ & $2.09 *$ & $3.25^{*}$ & $3.56^{*}$ & -0.70 & -2.13 & 1.07 & \\
\hline & (1.5) & (1.0) & $(1.2)$ & $(1.2)$ & $(0.7)$ & (1.5) & (1.2) & & $\begin{array}{c}(31.5 \\
)\end{array}$ & $(30.2)$ & $\begin{array}{c}(30.3 \\
)\end{array}$ & $\begin{array}{c}(28.3 \\
)\end{array}$ & $\begin{array}{c}(27.5 \\
)\end{array}$ & (28.3) & $(29.4)$ \\
\hline \multirow[t]{2}{*}{$5 \times 24$} & $\begin{array}{c}0.24 \\
*\end{array}$ & 0.05 & $\begin{array}{c}0.23 \\
*\end{array}$ & 0.07 & 0.06 & -0.14 & & $12 \times 16$ & $5.46^{*}$ & 0.63 & $3.41 *$ & $3.95 *$ & 0.81 & -2.66 & \\
\hline & (1.7) & $(1.0)$ & (1.4) & $(1.2)$ & $(0.7)$ & $(0.9)$ & (1.2) & & $\begin{array}{c}(33.3 \\
)\end{array}$ & $(29.6)$ & $\begin{array}{c}(32.5 \\
)\end{array}$ & $\begin{array}{c}(32.8 \\
)\end{array}$ & $\begin{array}{c}(32.5 \\
)\end{array}$ & $(28.0)$ & (31.4) \\
\hline \multirow[t]{2}{*}{$7 \times 14$} & 0.07 & -0.14 & $\begin{array}{c}0.23 \\
*\end{array}$ & $\begin{array}{c}0.33 \\
*\end{array}$ & -0.05 & 0.13 & & $3 \times 24$ & $5.03 *$ & $5.85^{*}$ & 2.29 & $7.57 *$ & 0.78 & $4.42 *$ & \\
\hline & (1.3) & $(0.9)$ & (1.5) & (1.4) & $(0.7)$ & (1.4) & $(1.2)$ & & $\begin{array}{c}(29.8 \\
)\end{array}$ & $(30.1)$ & $\begin{array}{c}(31.5 \\
)\end{array}$ & $\begin{array}{c}(32.0 \\
)\end{array}$ & $\begin{array}{c}(28.0 \\
)\end{array}$ & $(20.0)$ & $(28.6)$ \\
\hline S.E. (Sij) & 0.10 & 0.08 & 0.10 & 0.10 & 0.06 & 0.12 & & S.E. (Sij) & 1.03 & 1.66 & 1.23 & 1.32 & 1.23 & 1.47 & \\
\hline
\end{tabular}

*Significant at $\mathrm{P}=0.05$ level.

$\mathrm{E}_{1}$ and $\mathrm{E}_{4}-$ Non-ratoon early sown crop, $\mathrm{E}_{2}$ and $\mathrm{E}_{5}-$ Ratoon crop, $\mathrm{E}_{3}$ and $\mathrm{E}_{6}-$ Non-ratoon late sown crop 
The hybrid 1 x 14 (M $\quad$ x $\quad$ L) expressed significant positive sca effects for all four characters in most the environments. The hybrid 1 x 14 (M x L) involving both poor combinations for grain yield expressed significant positive sca effects in all the environments and also gave high per se. In general, most of the crosses showed no relationship between sca and per se. Inability of a single cross to rank uniformly in all the environments on the basis of per se performance as well as sca in different environmental situations has been reported by Sagar and Kapoor (1988). The hybrid 1 x 13 (81A x H90/4-5=HHB50, a released hybrid) involving $\mathrm{M} \times \mathrm{L}$ combining parents gave positive sca effects in three environments involving environments $\left(\mathrm{E}_{2}, \mathrm{E}_{4}, \mathrm{E}_{5}\right)$ during both the years for grain yield. Interestingly the parents 81A and H90/4-5 of HHB-50 also possessed regeneration potential. Besides grain yield it also gave high sca and per se for 500-grain weight. This confirms the superiority /good performance across the environments of this released (HHB-50 hybrid).

The hybrids $1 \times 13\left(81 \mathrm{~A}_{1} \times \mathrm{H} 90 / 4-5\right), 1 \times 14$ $\left(81 \mathrm{~A}_{1} \times \mathrm{H} 77 / 833-2\right), 9 \times 18\left(\mathrm{~Pb} 313 \mathrm{~B}_{2} \times\right.$

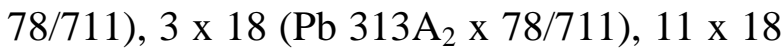
$\left(81 B_{4} \times 78 / 711\right), 12 \times 16\left(81 B_{5} \times\right.$ CSSC 46-2), $7 \times 21\left(81 B_{1} \times\right.$ ISK 48) showed significant positive sca effects for grain yield and one or more characters in most of the environments. The hybrids based on diverse male sterility systems A1, $A_{2}, A 3$, and A4 has significant sca effects for grain yield and some others characters indicating the a distinct advantage of these cytoplasm sources. Rai et al., (1996) reported that A2 and A3 source is highly unstable and is commercially in viable. However, A4 and $\mathrm{A}_{5}$ sources have been shown to be highly stable. Therefore, the results of this study on combining ability suggest that other than A1 source, A4 and A5 systems should provide a good opportunity to diversify the cytoplasmic base of pearl millet.
Based on the results obtained in the present study it can also be concluded that the hybrids based on $A_{1}, A_{2}$ and $A_{5}$ cytoplasms were superior to other cytoplasmic sources. The parents involved in these crosses were allowed to intermate among them for few generations to get desirable recombinants. The hybrids $1 \times 13\left(81 \mathrm{~A}_{1} \times \mathrm{H} 90 / 4-5\right), 1 \times 14$ $\left(81 \mathrm{~A}_{1} \times \mathrm{H} 77 / 833-2\right), 9$ x $13\left(313 \mathrm{~B}_{2}\right.$ x H90/4$5)$ and $12 \times 16\left(81 B^{5} \times\right.$ CSSC46-2) performed better on the basis of per se, sca effects including ratoon crop indicating their hope for sustainable dual purpose pearl millet production in harsh environments and can be recombined to develop base population for improvement for ratoonability by cyclic breeding.

\section{Acknowledgement}

Authors acknowledge the CCS Haryana Agricultural University to carry out this research work

\section{References}

Bhadalia, A.S., Dhedhi, K.K., Joshi, H.J. and Sorathiya, J.S. (2014). Combining ability studies through diallel analysis in pearl millet (Pennistum gluacum (L.) R. Br.) Internat.J.agri.Sci., 10(1): 57-60.

Bramel-Cox, P.J., Andrews, D.J., Bidinger, F.R. and Frey, K.J. (1984). A rapid method of evaluating growth rate in pearl millet and its weedy and wild relatives. Crop Sci. 24: 1187-1191.

Day,A.D., Thompson, R.K. and McCaughey, W.F. (1968). Effect of clipping on the performance of spring barley (Hordeum vulgare L. emend Lam.) seeded in October. Agron. J. 60: 11-12.

Donald, C.M. (1962). In search of yield. $J$. Australian Institute Agric. Sci. 28: 171178.

Federer, W.T. (1977). Experimental Design, Theory and Application. The Macmillon Co., New York. 
Kempthorne, O. (1957). An introduction to genetic statistics, John Wiley and Sons New York, USA.

Kumar, A, Kumar, R., Devvart and Dehinwal, A. K. (2017). Studies on heterosis involving diverse cytoplasmic male sterility systems in pearl millet. Journal of Pharmacognosy and Phytochemistry 6(6): 2056-2061

Kumar, A., Kumar, R., Devvart and Dehinwal, A.K. (2017). Combining ability for grain yield and its components involving alloplasmic isonuclear lines of pearl millet. International Journal of Current Microbiology and Applied Sciences 6(8): 554-61.

Kumar, R. and Sagar, P. (2015). Effect of cytoplasm on combining ability and dry fodder yield contributing traits in pearl millet [Pennisetumglaucum (1.) R. Br.] Forage res., 40 (4): 209-217.

Kumar, R., 2002. Studies on effect of cytoplasm on productivity traits and combining ability in direct sown and regenerated pearl millet (Pennisetum glaucum (L.) R. Br.). Ph.D. Dissertation, Haryana Agricultural University, Hisar.

Kumar, R., and P. Sagar. 2010. Effect of cytoplasm on combining ability and yield attributes in pearl millet [Pennisetum glaucum (L.) R. Br.]. Indian J. Genet., 70: 247-256.

Kumar, R., Sagar P. and Behl, R.K. (2005). Evaluation of regeneration potential in pearl millet. Acta Agronomica Hungarica, 53(4) 423-432.

Kumar, S., Chahal, G.S. and Virk, D.S. (1996). Combining ability of diverse male sterility sources in pearl millet. Crop Improv. 23(1): 151-154.

Mangat, B.K., Virk D.S. and Randhawa, H.S. (1998). Comparative performance of near-isonuclear hybrids of pearl millet for grain yield and some morphological traits. Crop Improv. 25 (1): 91-95.

Mangath, K.S. (1987). Hybrid vigour for green fodder and grain yield in white grained pearl millet. Indian J. Hered. 19 (1-2): 10-15.

Mungra, K, S., Dobariya, K.L., Sapodiya, M.H. and Vavdiya. P.A. (2015). Combining ability and gene action for grain yield and its component traits in pearl millet. (Pennistum gluacum (L.) R. Br.) Electronic J.Pl.Br. 6(1): 66-73

Rafiq, S.M., Sunil, B. and Prasada Rao. U. (2016). Combining ability studies using diverse cytoplasm for grain yield and its components in pearl millet. (Pennistum gluacum (L.) R. Br.) Plant archives., 16(2): 588-593

Rai, K.N., Virk, D.S., Harinarayana, G., and Rao A.S. (1996). Stability of male sterility sources and fertility restoration of their hybrids in pearl millet. Plant Breed., 115: 494-500.

Renner, O. and Kupper, W. (1921). Artkreuzungen in der gattung Epitobium. Ber Dtsch Botan Ges., 39: 201-206.

Sagar, Prem and Kapoor, R.L. (1988). Genetics of grain yield and drought index in pearl millet (Pennisetum typhoides) under moisture stress. Indian J. Agric. Sci. 58 (2): 104-109.

Sharma, S.C., Gupta, V.P. and Satija, D.R. (1996). Components of variance in line $x$ tester mating as influenced by the choice of testers on the basis of diallel cross analysis in pearl millet. Crop Improv. 23 (1): 121-125.

Skorda, A. (1977). Effect of clipping on forage, hay and grain production from barley, wheat and triticale. Proc. Fourth Regional Winter Cereals Workshop, 11: Amman, Jordan

Sprague, G.F. and Tatum, L.A. (1942). General vs specific combining ability in single crosses of corn. J. American Society Agron. 34: 923-932. 
Sudhir Sharma (2005). Combining ability, Heterosis and Stability analysis of different Cytoplasmic sources in pearl millet (Pennisetum glaucum (L.) R. Br.). Ph.D. Dissertation, Haryana Agricultural University, Hisar.

Sudhir Sharma, Kumar R. and Yadav, H.P. (2019). Combining ability of new male sterile lines of diverse sources in pearl millet for yield and forage components. Journal of Pharmacognosy and Phytochemistry, 8(1): 533-536.

Sudhir Sharma, Yadav, H.P., Kumar R. and DevVart (2019). Genetic analysis for micronutrient and grain yield in relation to diverse sources of cytoplasm in pearl millet (Pennistum gluacum (L.) R. Br.) Internal Journal of current microbiology and applied Sciences; 8(1): 613-624.

Tomer, P.S. and Saini, S.L. (1979). Studies on ratoonability in early sown pearl millet. Haryana Agricultural University J. Res. IX (2): 138-141.

Virk, D.S. and Brar, J.S. (1993). Assessment of cytoplasmic differences of nearisonuclear male-sterile lines in pearl millet. Theor. Appl. Genet. 87: 106-112.

Yadav, O.P. (1999). Heterosis and combining ability in relation to cytoplasmic diversity in pearl millet. Indian $J$. Genet. 59(4): 445-450.

Yadav, O.P., Weltzien, E.R, Mahalakshmi, V. and Bidinger, F.R. (2000). Combining ability of pearl millet landraces originating from afid area of Rajasthan. Indian J. Genet. 60(1): 45-53.

Young, J.B. and Virmani, S.S. 1990. Effects of cytoplasm on heterosis and combining ability for agronomic traits in rice (Oryza sativa L.). Euphytica, 48: $177-188$.

\section{How to cite this article:}

Ramesh Kumar, Sudhir Sharma, Prem Sagar and Ashok Kumar. 2020. Genetic Analysis of Grain Yield and Its Attributes Using Diverse Systems of Male Sterile Lines and Pollinators in Direct Sown and Ratooned Pearl Millet. Int.J.Curr.Microbiol.App.Sci. 9(02): 486-499. doi: https://doi.org/10.20546/ijcmas.2020.902.060 